\title{
Endovascular management of cerebral aneurysms
}

\author{
Felipe C. Albuquerque, M.D. \\ Division of Neurological Surgery, Barrow Neurological Institute, St. Joseph's Hospital and Medical \\ Center, Phoenix, Arizona
}

The endovascular management of cerebral aneurysms continues to evolve. Novel technologies have both expanded the range of available treatments and improved patient outcomes. In light of the results of the International Subarachnoid Aneurysm Trial, controversy exists as to the optimal management of cerebral aneurysms. ${ }^{1}$ In this issue of Neurosurgical Focus, the contributors explore several recent advances in the endovascular treatment of these lesions, and they also relate the history and evolving strategies of endovascular aneurysm management.

Specifically, in this issue the investigators describe the use of several novel endovascular devices, including selfexpanding coated coils and flexible stents uniquely designed for navigation in the central nervous system. Also addressed are specific aneurysm types and clinical scenarios, such as distal vertebral artery dissections and aneurysmal dolichoectasia of the posterior circulation. The endovascular management and pathophysiological findings in patients with these lesions are reviewed. Finally, in this issue the authors provide a historical perspective on the embolization of cerebral aneurysms in two articles focusing on the evolution of interventional techniques.

Without question, endovascular therapy has revolutionized the management of cerebrovascular disease. The rapidity of the evolution of these devices and procedures has broadened the clinical efficacy and reach of neurosurgeons and neuroradiologists alike. For endovascular specialists, the future is now.

\section{Reference}

1. International Subarachnoid Aneurysm Trial (ISAT) Collaborative Group: International Subarachnoid Aneurysm Trial (ISAT) of neurosurgical clipping versus endovascular coiling in 2143 patients with ruptured intracranial aneurysms: a randomised trial. Lancet 360:1267-1274, 2002 